\title{
High-energy behavior of the nuclear symmetry potential in asymmetric nuclear matter
}

\author{
Lie-Wen Chen, ${ }^{1,2}$ Che Ming Ko, ${ }^{3}$ and Bao-An Li ${ }^{4}$ \\ ${ }^{1}$ Institute of Theoretical Physics, Shanghai Jiao Tong University, Shanghai 200240, China \\ ${ }^{2}$ Center of Theoretical Nuclear Physics, National Laboratory of Heavy Ion Accelerator, Lanzhou 730000, China \\ ${ }^{3}$ Cyclotron Institute and Physics Department, Texas A\&M University, College Station, Texas 77843-3366, USA \\ ${ }^{4}$ Department of Chemistry and Physics, Arkansas State University, Jonesboro, Arkansas 72467-0419, USA
}

(Received 23 August 2005; published 15 December 2005)

\begin{abstract}
Using the relativistic impulse approximation with empirical $N N$ scattering amplitude and the nuclear scalar and vector densities from the relativistic mean-field theory, we evaluate the Dirac optical potential for neutrons and protons in asymmetric nuclear matter. From the resulting Schrödinger-equivalent potential, the high-energy behavior of the nuclear symmetry potential is studied. We find that the symmetry potential at fixed baryon density is essentially constant once the nucleon kinetic energy is greater than about $500 \mathrm{MeV}$. Moreover, for such a high-energy nucleon, the symmetry potential is slightly negative below a baryon density of about $\rho=0.22 \mathrm{fm}^{-3}$ and then increases almost linearly to positive values at high densities. Our results thus provide an important constraint on the energy and density dependence of nuclear symmetry potential in asymmetric nuclear matter.
\end{abstract}

DOI: 10.1103/PhysRevC.72.064606

PACS number(s): 21.65.+f, 21.30.Fe, 24.10.Jv

\section{INTRODUCTION}

Recently, there is renewed interest in the isovector part of the nucleon mean-field potential, i.e., the nuclear symmetry potential, in isospin asymmetric nuclear matter [1-16]. Knowledge on the symmetry potential is important for understanding not only the structure of radioactive nuclei and the reaction dynamics induced by rare isotopes but also many critical issues in astrophysics. In addition to depending on the nuclear density, the symmetry potential also depends on the momentum or energy of a nucleon. Various microscopic and phenomenological models, such as the relativistic Dirac-BruecknerHartree-Fock (DBHF) $[2,8,9,13,15]$ and the nonrelativistic Brueckner-Hartree-Fock (BHF) [1,14] approaches, the relativistic mean-field theory based on nucleon-meson interactions [12], and the nonrelativistic mean-field theory based on Skyrme-like interactions $[3,11]$, have been used to study the symmetry potential. However, the results predicted by these models vary widely. In particular, whereas most models predict a decreasing symmetry potential with increasing nucleon momentum albeit at different rates, a few nuclear effective interactions used in some of the models lead to the opposite conclusion. Thus, any constraint on the momentum and density dependence of the symmetry potential is very useful.

In the optical model based on the Dirac phenomenology, elastic nucleon-nucleus scattering is described by the Dirac equation for the motion of a nucleon in a relativistic potential. For spherical nuclei, good agreements with experimental data were obtained in the relativistic approach with a scalar potential (nucleon scalar self-energy) and the zeroth component of a vector potential (nucleon vector self-energy), whereas the standard nonrelativistic optical model using the Schrödinger equation failed to describe simultaneously all experimental observables [17]. Motivated by the success of the Dirac phenomenology, a microscopic relativistic model based on the impulse approximation (RIA) [18-21] was developed, and it was able to fit the data from $p+{ }^{40} \mathrm{Ca}$ and $p+{ }^{208} \mathrm{~Pb}$ elastic scattering at nucleon energies of both 500 and
$800 \mathrm{MeV}$ very well. A nice feature of the RIA is that it permits very little phenomenological freedom in deriving the Dirac optical potential in nuclear matter. The basic ingredients in this method are the free invariant nucleon-nucleon $(N N)$ scattering amplitude and the nuclear scalar and vector densities in nuclear matter. This is in contrast to the relativistic DBHF approach, where different approximation schemes and methods have been introduced for determining the Lorentz and isovector structure of the nucleon self-energy $[2,8,9,13,15]$.

In the present work, we evaluate the Dirac optical potential for neutrons and protons in asymmetric nuclear matter based on the relativistic impulse approximation by using the $N N$ scattering amplitude determined by McNeil, Ray, and Wallace [22], which has been shown to be valid for nucleons with kinetic energy greater than about $300 \mathrm{MeV}$. The highenergy behavior of the nuclear symmetry potential from the resulting Schrödinger-equivalent potential is then investigated without adjustable parameters. We find that the nuclear symmetry potential at fixed density becomes almost constant for nucleon kinetic energy greater than about $500 \mathrm{MeV}$. For such high-energy nucleons, the nuclear symmetry potential is further found to be weakly attractive below a nuclear density of about $\rho=0.22 \mathrm{fm}^{-3}$ but to become increasingly repulsive when the nuclear density increases.

The article is organized as follows. In Sec. II, we briefly review the relativistic impulse approximation for nuclear optical potential and the relativistic mean-field model for nuclear scalar and vector densities. Results on the relativistic nuclear optical potential and the nuclear symmetry potential in asymmetric nuclear matter are presented in Sec. III. A short summary is then given in Sec. IV.

\section{NUCLEAR OPTICAL POTENTIAL}

\section{A. Relativistic impulse approximation}

Many theoretical studies have suggested that nucleonnucleus scattering at sufficient high energy can be viewed as the 
projectile nucleon being scattered from each of the nucleons in the target nucleus. One thus can describe the process by using the $N N$ scattering amplitude and the ground-state nuclear density distribution of the target nucleus. For the Lorentz-invariant $N N$ scattering amplitude, it can be written as

$$
\begin{aligned}
\widehat{F}= & F_{S}+F_{V} \gamma_{1}^{\mu} \gamma_{2 \mu}+F_{T} \sigma_{1}^{\mu v} \sigma_{2 \mu \nu} \\
& +F_{P} \gamma_{1}^{5} \gamma_{2}^{5}+F_{A} \gamma_{1}^{5} \gamma_{1}^{\mu} \gamma_{2}^{5} \gamma_{2 \mu}
\end{aligned}
$$

in terms of the scalar $F_{S}$, vector $F_{V}$, tensor $F_{T}$, pseudoscalar $F_{P}$, and axial vector $F_{A}$ amplitudes. In the above, subscripts 1 and 2 distinguish Dirac operators in the spinor space of the two scattering nucleons and $\gamma$ 's are $\gamma$ matrices. The five complex amplitudes $F_{S}, F_{V}, F_{T}, F_{P}$, and $F_{A}$ depend on the squared momentum transfer $\mathbf{q}^{2}$ and the invariant energy of the scattering nucleon pair, and they were determined in Ref. [22] directly from the $N N$ phase shifts that were used to parametrize the $N N$ scattering data. For a spin-saturated nucleus, only the scalar $\left(F_{S}\right)$ and the zeroth component of the vector $\left(F_{V} \gamma_{1}^{0} \gamma_{2}^{0}\right)$ amplitudes dominate the contribution to the optical potential. In the relativistic impulse approximation, the optical potential in momentum space is thus obtained by multiplying each of these two amplitudes with corresponding momentum-space nuclear scalar $\tilde{\rho}_{S}(\mathbf{q})$ and vector $\tilde{\rho}_{V}(\mathbf{q})$ densities, i.e.,

$$
\tilde{U}_{\mathrm{opt}}(\mathbf{q})=\frac{-4 \pi i p_{\mathrm{lab}}}{M}\left[F_{S}(q) \tilde{\rho}_{S}(\mathbf{q})+\gamma_{0} F_{V}(q) \tilde{\rho}_{V}(\mathbf{q})\right],
$$

where $p_{\text {lab }}$ and $M$ are, respectively, the laboratory momentum and mass of the incident nucleon. The optical potential in coordinator space is then given by the Fourier transformation of $\tilde{U}_{\text {opt }}(\mathbf{q})$, similar to the " $t \rho$ " approximation used in nonrelativistic impulse approximation [22].

The $\mathbf{q}$ dependence of the relativistic $N N$ amplitude is important for calculating observables of a nucleon scattering off finite nuclei within the Dirac phenomenology. In the present work, we are interested in the strength of the Dirac optical potential of nucleons in infinite nuclear matter. Because the scalar and vector densities in coordinate space are constant in infinite nuclear matter, they are delta functions in momentum space, i.e., $\sim \delta^{(3)}(\mathbf{q})$. In this case, only the forward $N N$ scattering amplitudes, i.e., $F_{S 0} \equiv F_{S}(q=0)$ and $F_{V 0} \equiv F_{V}(q=0)$, contribute to the Fourier transform of Eq. (2), and the nuclear coordinate-space optical potential takes the simple form [18]:

$$
U_{\mathrm{opt}}=\frac{-4 \pi i p_{\mathrm{lab}}}{M}\left[F_{S 0} \rho_{S}+\gamma_{0} F_{V 0} \rho_{V}\right],
$$

where $\rho_{S}$ and $\rho_{V}$ are, respectively, the spatial scalar and vector densities of an infinite nuclear matter.

The optical potential $U_{\text {opt }}$ is a $4 \times 4$ matrix in the Dirac spinor space of the projectile nucleon and includes the following scalar $U_{S}^{\text {tot }}$ and vector $U_{0}^{\text {tot }}$ pieces:

$$
U_{\mathrm{opt}}=U_{S}^{\mathrm{tot}}+\gamma_{0} U_{0}^{\mathrm{tot}}
$$

Because $U_{S}^{\text {tot }}$ and $U_{0}^{\text {tot }}$ are generally complex, they can be expressed in terms of their real and imaginary parts, i.e.,

$$
U_{S}^{\mathrm{tot}}=U_{S}+i W_{S}, \quad U_{0}^{\mathrm{tot}}=U_{0}+i W_{0},
$$

\section{B. Nuclear scalar densities}

To evaluate the RIA optical potential for neutrons and protons, we need the values for the isospin-dependent $F_{S 0}$ and $F_{V 0}$, which can be found in Ref. [22], as well as the scalar and vector densities for neutrons and protons. For the latter, we determine them using the relativistic mean-field (RMF) theory [23] with a Lagrangian density that includes the nucleon field $\psi$, the isoscalar-scalar meson field $\sigma$, the isoscalar-vector meson field $\omega$, the isovector-vector meson field $\rho$, and the isovector-scalar meson field $\delta$, i.e.,

$$
\begin{aligned}
\mathcal{L}(\psi, \sigma, \omega, \rho, \delta)= & \bar{\psi}\left[\gamma_{\mu}\left(i \partial^{\mu}-g_{\omega} \omega^{\mu}\right)-\left(M-g_{\sigma} \sigma\right)\right] \psi \\
& +\frac{1}{2}\left(\partial_{\mu} \sigma \partial^{\mu} \sigma-m_{\sigma}^{2} \sigma^{2}\right)-\frac{1}{4} \omega_{\mu \nu} \omega^{\mu \nu} \\
& +\frac{1}{2} m_{\omega}^{2} \omega_{\mu} \omega^{\mu}-\frac{1}{3} b_{\sigma} M\left(g_{\sigma} \sigma\right)^{3}-\frac{1}{4} c_{\sigma}\left(g_{\sigma} \sigma\right)^{4} \\
& +\frac{1}{4} c_{\omega}\left(g_{\omega}^{2} \omega_{\mu} \omega^{\mu}\right)^{2}+\frac{1}{2}\left(\partial_{\mu} \delta \partial^{\mu} \delta-m_{\delta}^{2} \delta^{2}\right) \\
& +\frac{1}{2} m_{\rho}^{2} \rho_{\mu} \rho^{\mu}-\frac{1}{4} \rho_{\mu \nu} \rho^{\mu \nu}+\frac{1}{2}\left(g_{\rho}^{2} \rho_{\mu} \rho^{\mu}\right) \\
& \times\left(\Lambda_{S} g_{\sigma}^{2} \sigma^{2}+\Lambda_{V} g_{\omega}^{2} \omega_{\mu} \omega^{\mu}\right) \\
& -g_{\rho} \rho_{\mu} \bar{\psi} \gamma^{\mu} \tau \psi+g_{\delta} \delta \bar{\psi} \tau \psi,
\end{aligned}
$$

where the antisymmetric field tensors $\omega_{\mu \nu}$ and $\rho_{\mu \nu}$ are given by $\omega_{\mu \nu} \equiv \partial_{\nu} \omega_{\mu}-\partial_{\mu} \omega_{\nu}$ and $\rho_{\mu \nu} \equiv \partial_{\nu} \rho_{\mu}-\partial_{\mu} \rho_{\nu}$, respectively, and the symbols used in Eq. (6) have their usual meanings. The above Lagrangian density is general and allows us to use many presently popular parameter sets. In the present work, we use three typical parameter sets, namely the very successful NL3 model [24], the Z271v model which was used to study the neutron skin of heavy nuclei and the properties of neutron stars [25], and the HA model, which includes the isovector-scalar meson field $\delta$ and fits successfully some results calculated with more microscopic DBHF approach [26].

In Fig. 1, we show the neutron and proton scalar densities $\rho_{S}$ as functions of the baryon density $\rho_{B}$ (vector density in the static infinite nuclear matter) in nuclear matter with isospin asymmetry $\alpha=0$ and 0.5 for parameter sets NL3, Z271v, and HA. The isospin asymmetry is defined as $\alpha=\left(\rho_{n}-\rho_{p}\right) / \rho_{B}$ with $\rho_{B}=\rho_{n}+\rho_{p}$ and $\rho_{n}$ and $\rho_{p}$ denoting the neutron and proton densities, respectively. It is seen that the neutron scalar

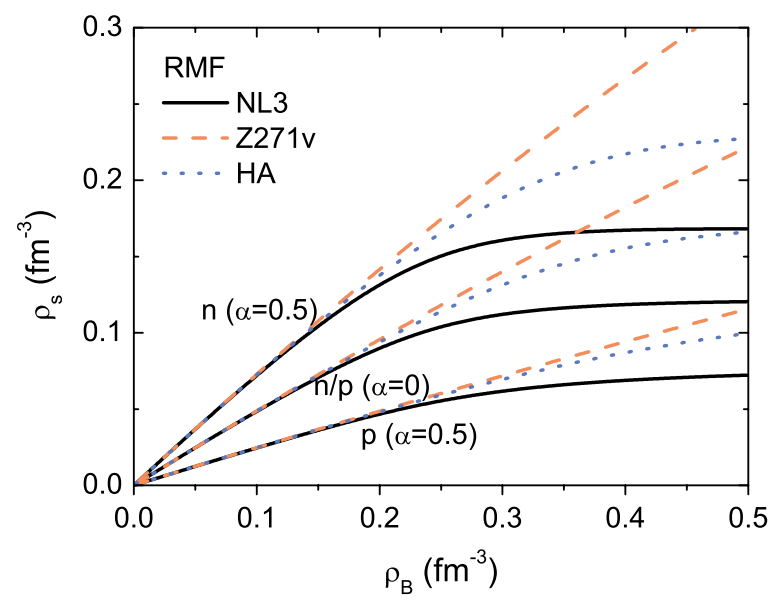

FIG. 1. (Color online) Neutron and proton scalar densities as functions of baryon density in nuclear matter with isospin asymmetry $\alpha=0$ and 0.5 for the parameter sets NL3, Z271v, and HA. 


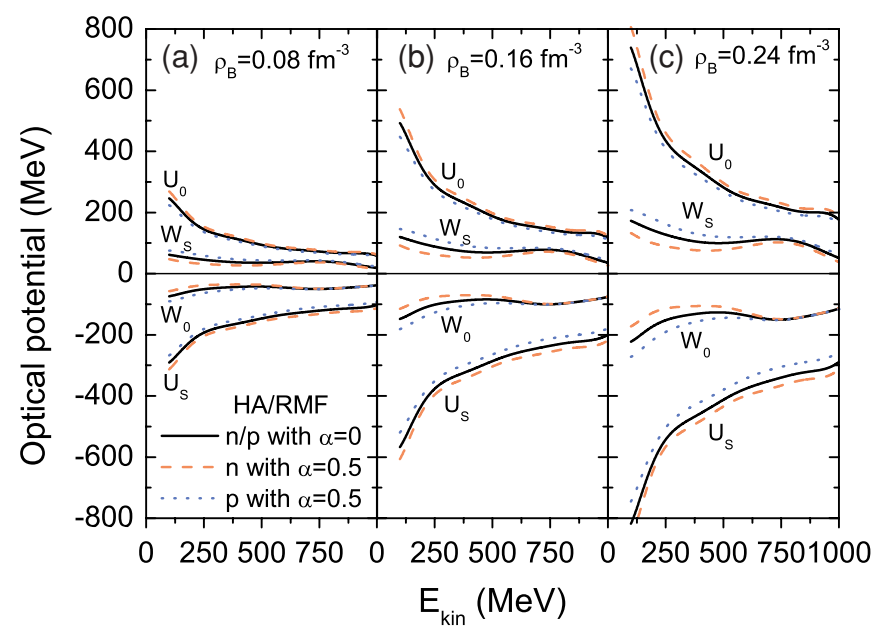

FIG. 2. (Color online) Energy dependence of real and imaginary parts of the scalar and vector potentials for neutrons and protons in nuclear matter with isospin asymmetry $\alpha=0$ and 0.5 for the parameter set HA.

density is larger than that of the proton at a fixed baryon density in neutron-rich nuclear matter. Although results for different parameter sets are almost the same at lower baryon densities, they become different when $\rho_{B} \gtrsim 0.25 \mathrm{fm}^{-3}$ with Z271v giving a larger and NL3 a smaller $\rho_{S}$ than that from the parameter set HA. The real and imaginary parts of the scalar potential at higher baryon densities thus depend on the interactions used in evaluating the nucleon scalar density and have, therefore, large uncertainties.

\section{RESULTS}

\section{A. Relativistic nuclear optical potential}

With neutron and proton scalar densities obtained from the RMF theory for the parameter set HA, we have studied both the energy and density dependence of the real and imaginary parts of the scalar and vector potentials for neutrons and protons in nuclear matter with isospin asymmetry $\alpha=0$ and 0.5 . In Fig. 2, the resulting energy dependence is shown for the three nucleon densities $\rho_{B}=0.08 \mathrm{fm}^{-3}$ [panel (a)], $0.16 \mathrm{fm}^{-3}$ [panel (b)], and $0.24 \mathrm{fm}^{-3}$ [panel (c)]. For all densities, the optical potential shows a strong energy dependence below $300 \mathrm{MeV}$, where it is known that the influences because of ambiguities in the relativistic form of the $N N$ interaction, the exchange contribution, and the medium modification because of Pauli blocking are important. The low-energy behavior of the optical potential can in principle be studied in the generalized relativistic impulse approximation based on the relativistic meson-exchange model of nuclear force and using the complete set of Lorentz-invariant $N N$ amplitudes [27-31]. Because many theoretical studies have shown that data on elastic nucleon-nucleus scattering can be reproduced by using above optical potential when the nucleon kinetic energy is greater than about $300 \mathrm{MeV}$ and that this optical potential also agrees very well with that extracted from phenomenological analysis of the nucleon-nucleus scattering data [18-20,32],

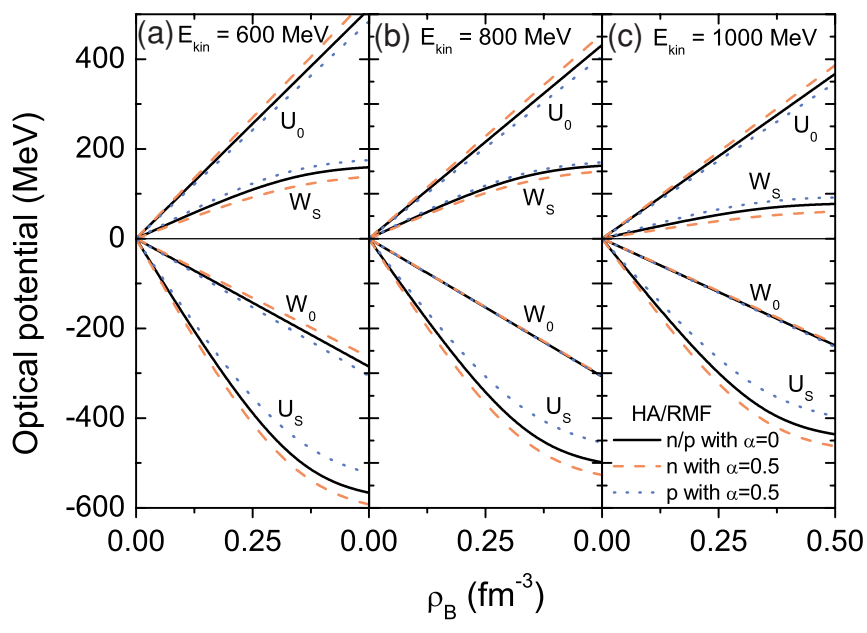

FIG. 3. (Color online) Density dependence of the real and imaginary parts of the scalar and vector potentials for neutrons and protons in nuclear matter with isospin asymmetry $\alpha=0$ and 0.5 for the parameter set HA.

we thus in the present work focus on the higher-energy behavior of the isospin-dependent optical potential. As shown in Fig. 2, for all three densities considered here, there is a systematic difference or isospin splitting in the optical potentials for protons and neutrons in asymmetric nuclear matter. Specifically, the neutron exhibits a stronger real but weaker imaginary scalar and vector potentials in neutron-rich nuclear matter. Furthermore, both the proton and neutron optical potentials become stronger with increasing density.

The density dependence of the real and imaginary parts of the scalar and vector potentials for neutrons and protons in nuclear matter with isospin asymmetry $\alpha=0$ and 0.5 obtained with the parameter set HA is shown more explicitly in Fig. 3 for the three nucleon kinetic energies of $E_{\text {kin }}=600 \mathrm{MeV}$ [panel (a)], $800 \mathrm{MeV}$ [panel (b)], and $1000 \mathrm{MeV}$ [panel (c)]. An isospin splitting of the nucleon optical potential in asymmetric nuclear matter is again clearly seen.

\section{B. Nuclear symmetry potential}

From the Dirac optical potential, a Schrödinger-equivalent potential (SEP) of the following form is usually introduced [33,34]:

$$
U_{\mathrm{SEP}}=U_{S}^{\mathrm{tot}}+U_{0}^{\mathrm{tot}}+\frac{1}{2 M}\left(U_{S}^{\mathrm{tot} 2}-U_{0}^{\mathrm{tot} 2}\right)+\frac{U_{0}^{\mathrm{tot}}}{M} \varepsilon,
$$

where $\varepsilon$ is the nucleon kinetic energy. Using the SEP in the Schrödinger equation gives the same bound-state energy eigenvalues and elastic phase shifts as the solution of the upper component of the Dirac spinor in the Dirac equation using corresponding Dirac optical potential. The real part of the SEP is then given by the following:

$$
\operatorname{Re}\left(U_{\mathrm{SEP}}\right)=U_{S}+U_{0}+\frac{1}{2 M}\left[U_{S}^{2}-W_{S}^{2}-\left(U_{0}^{2}-W_{0}^{2}\right)\right]+\frac{U_{0}}{M} \varepsilon .
$$




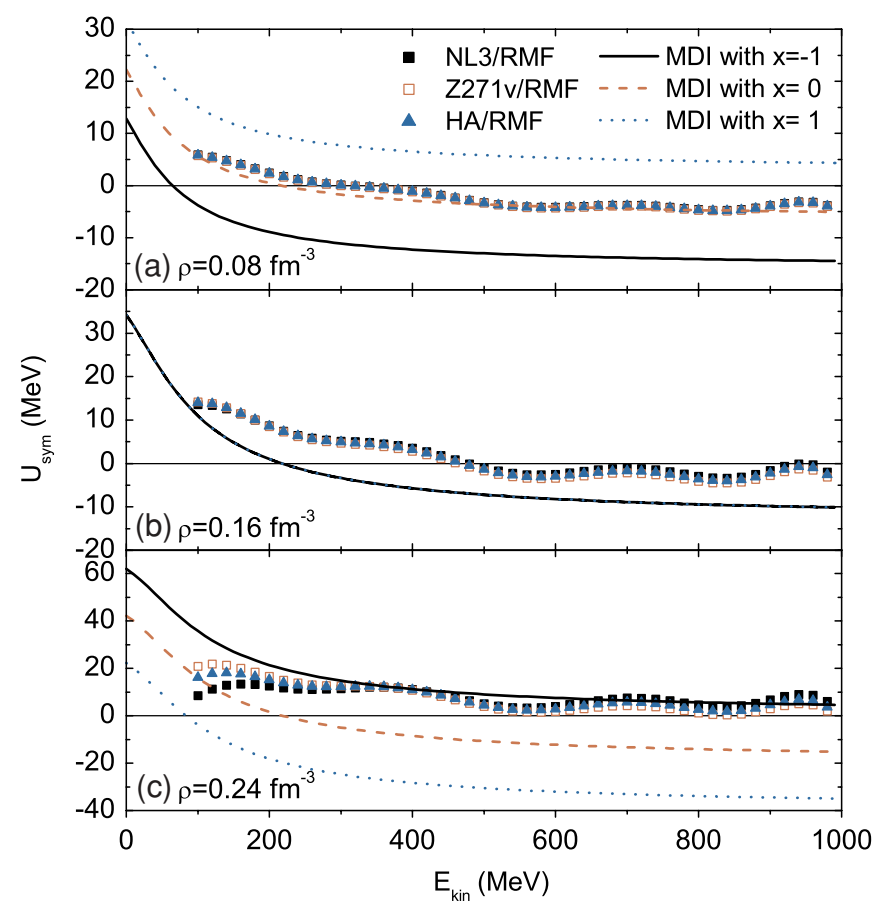

FIG. 4. (Color online) Energy dependence of the nuclear symmetry potential using the parameter sets NL3, Z271v, and HA as well as from the phenomenological interaction MDI with $x=-1,0$, and 1 at fixed baryon densities of $\rho_{B}=0.08 \mathrm{fm}^{-3}$ (a), $0.16 \mathrm{fm}^{-3}$ (b), and $0.24 \mathrm{fm}^{-3}(\mathrm{c})$.

This equation corresponds to the nuclear mean-field potential in nonrelativistic models $[15,35]$ and allows us to obtain the following nuclear symmetry potential, i.e., the so-called Lane potential [36]:

$$
U_{\text {sym }}=\frac{\operatorname{Re}\left(U_{\mathrm{SEP}}\right)_{n}-\operatorname{Re}\left(U_{\mathrm{SEP}}\right)_{p}}{2 \alpha},
$$

where $\operatorname{Re}\left(U_{\mathrm{SEP}}\right)_{n}$ and $\operatorname{Re}\left(U_{\mathrm{SEP}}\right)_{p}$ represent, respectively, the real part of the SEP for the neutron and proton.

In Fig. 4, we show the energy dependence of the nuclear symmetry potential for the parameter sets NL3, Z271v, and HA at fixed baryon densities of $\rho_{B}=0.08 \mathrm{fm}^{-3}$ [panel (a)], $0.16 \mathrm{fm}^{-3}$ [panel (b)], and $0.24 \mathrm{fm}^{-3}$ [panel (c)]. It is seen that all three parameter sets give a similar nuclear symmetry potential for a nucleon at kinetic energies higher than about $300 \mathrm{MeV}$, i.e., it first decreases with nucleon kinetic energy and then becomes essentially constant when the nucleon kinetic energy is above about $500 \mathrm{MeV}$. Specifically, the nuclear symmetry potential starts from about $0 \mathrm{MeV}$ at a lower density of $\rho_{B}=$ $0.08 \mathrm{fm}^{-3}$ (about half of nuclear saturated density), $4.8 \mathrm{MeV}$ at a normal nuclear matter density $\left(\rho_{B}=0.16 \mathrm{fm}^{-3}\right)$, and $12 \mathrm{MeV}$ at a higher density of $\rho_{B}=0.24 \mathrm{fm}^{-3}$ (about 1.5 time nuclear saturated density) and then saturates to about $-3.8 \pm 0.5 \mathrm{MeV},-1.8 \pm 1.7 \mathrm{MeV}$, and $5.3 \pm 3.8 \mathrm{MeV}$, respectively, when the nucleon kinetic energy is greater than about $500 \mathrm{MeV}$. The uncertainties in the saturated values simply reflect the variation in the energy dependence of the symmetry potential at high energies.

For comparison, we also show in Fig. 4 results from the phenomenological parametrization of the momentum- dependent nuclear mean-field potential, i.e., MDI interaction with $x=-1,0$, and 1 . In the MDI interaction, the single nucleon potential in asymmetric nuclear matter with isospin asymmetry $\alpha$ is expressed by the following $[3,5,6,10]$ :

$$
\begin{aligned}
U(\rho, \alpha, \mathbf{p}, \tau, \mathbf{r})= & \left(-95.98-x \frac{2 B}{\sigma+1}\right) \frac{\rho_{\tau^{\prime}}}{\rho_{0}} \\
& +\left(-120.57+x \frac{2 B}{\sigma+1}\right) \frac{\rho_{\tau}}{\rho_{0}}+B\left(\frac{\rho}{\rho_{0}}\right)^{\sigma} \\
& \times\left(1-x \alpha^{2}\right)-8 \tau x \frac{B}{\sigma+1} \frac{\rho^{\sigma-1}}{\rho_{0}^{\sigma}} \alpha \rho_{\tau^{\prime}} \\
& +\frac{2 C_{\tau, \tau}}{\rho_{0}} \int d^{3} \mathbf{p}^{\prime} \frac{f_{\tau}\left(\mathbf{r}, \mathbf{p}^{\prime}\right)}{1+\left(\mathbf{p}-\mathbf{p}^{\prime}\right)^{2} / \Lambda^{2}} \\
& +\frac{2 C_{\tau, \tau^{\prime}}}{\rho_{0}} \int d^{3} \mathbf{p}^{\prime} \frac{f_{\tau^{\prime}}\left(\mathbf{r}, \mathbf{p}^{\prime}\right)}{1+\left(\mathbf{p}-\mathbf{p}^{\prime}\right)^{2} / \Lambda^{2}} .
\end{aligned}
$$

In the above $\tau=1 / 2(-1 / 2)$ for neutrons (protons) and $\tau \neq \tau^{\prime} ; \sigma=4 / 3$, and $f_{\tau}(\mathbf{r}, \mathbf{p})$ is the phase-space distribution function at coordinate $\mathbf{r}$ and momentum $\mathbf{p}$. The parameters $B, C_{\tau, \tau}, C_{\tau, \tau^{\prime}}$, and $\Lambda$ were determined by fitting the momentum dependence of $U(\rho, \alpha, \mathbf{p}, \tau, \mathbf{r})$ to that predicted by the Gogny Hartree-Fock and/or the BHF calculations [1], the saturation properties of symmetric nuclear matter and the symmetry energy of $31.6 \mathrm{MeV}$ at normal nuclear matter density $\rho_{0}=0.16 \mathrm{fm}^{-3}$ [3]. The incompressibility $K_{0}$ of symmetric nuclear matter at $\rho_{0}$ is set to be $211 \mathrm{MeV}$. The different $x$ values in the MDI interaction are introduced to vary the density dependence of the nuclear symmetry energy while keeping other properties of the nuclear EOS fixed [10]. We note that the energy dependence of the symmetry potential from the MDI interaction is consistent with the empirical Lane potential at normal nuclear matter density and low nucleon energies [4] and has been used in the transport model for studying isospin effects in intermediate-energy heavy-ion collisions induced by neutron-rich nuclei $[5,6,10]$.

It is seen from Fig. 4 that results from RIA at lower density of $\rho=0.08 \mathrm{fm}^{-3}$ are comparable to those from the MDI interaction with $x=0$, whereas at higher baryon density of $\rho_{B}=0.24 \mathrm{fm}^{-3}$ they are comparable to those from the MDI interaction with $x=-1$. At normal nuclear matter density, the MDI interaction, which gives same results for different $x$ values by construction, is seen to lead to smaller nuclear symmetry potential at high nucleon kinetic energies compared with present results from the RIA based on empirical $N N$ scattering amplitude and the nuclear scalar density from the relativistic mean-field theory. We note that our results agree surprisingly well with those of DBHF by Fuchs et al. [8,37].

For the density dependence of the nuclear symmetry potential using the parameter sets NL3, Z271v, and HA at a fixed high nucleon kinetic energy of $800 \mathrm{MeV}$, it is shown in Fig. 5 together with corresponding results from the MDI interaction with $x=-1,0$, and 1 . It is clearly seen that the nuclear symmetry potential from all parameter sets NL3, $\mathrm{Z} 271 \mathrm{v}$, and HA changes from negative to positive values at a fixed baryon density of about $\rho_{B}=0.22 \mathrm{fm}^{-3}$ and then increases almost linearly with baryon density. Furthermore, the nuclear symmetry potential depends very little on the choice of the parameter sets NL3, Z271v, and HA. At such high nucleon 


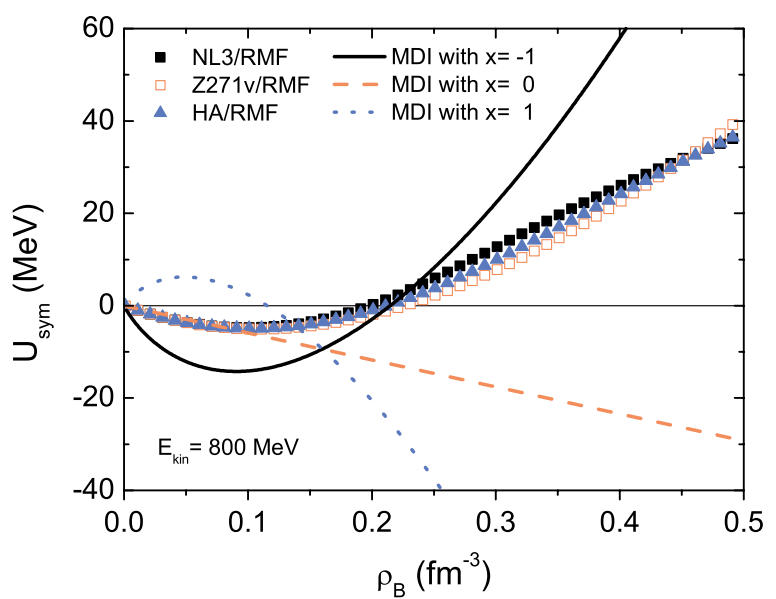

FIG. 5. (Color online) Density dependence of the nuclear symmetry potential using the parameter sets NL3, Z271v, and HA as well as from the MDI interaction with $x=-1,0$, and 1 at a fixed nucleon kinetic energy of $800 \mathrm{MeV}$.

kinetic energy, the nuclear symmetry potential from the MDI interaction with $x=0$ reproduces nicely the results from the RIA when $\rho_{B} \lesssim 0.1 \mathrm{fm}^{-3}$ as in its energy dependence at low densities shown in Fig. 4. The two differ strongly, however, at high densities. The MDI interaction with both $x=-1$ and 1 , conversely, show very different density dependence from present RIA results.

\section{SUMMARY}

We have evaluated the Dirac optical potential for neutrons and protons in asymmetric nuclear matter based on the relativistic impulse approximation with empirical $N N$ scattering amplitude and the scalar and vector densities from the relativistic mean-field theory. We find that the nuclear symmetry potential derived from the resulting Schrödinger-equivalent potential is not very sensitive to the parameter sets used in the relativistic mean-field calculation, particularly at low densities and high nucleon energies, although the latter give very different nuclear scalar densities at high baryon densities in both symmetric and asymmetric nuclear matters. Furthermore, the nuclear symmetry potential at a fixed density becomes almost constant when the nucleon kinetic energy is greater than about $500 \mathrm{MeV}$. For such high energy nucleon, our study shows that the density dependence of its nuclear symmetry potential is weakly attractive at low densities but become increasingly repulsive as nuclear density increases. Results presented in present study thus provide important constraints on the high energy behavior of the nuclear symmetry potential in asymmetric nuclear matter, which is an important input to the isospin-dependent transport model $[5,12]$ in studying heavyion collisions induced by radioactive nuclei at intermediate and high energies. They are also useful in future studies that extend the Lorentz-covariant transport model $[38,39]$ to include explicitly the isospin degrees of freedom.

\section{ACKNOWLEDGMENTS}

This work was supported in part by the National Natural Science Foundation of China under grants 10105008 and 10575071 (L.W.C.), the U.S. National Science Foundation under grant PHY-0457265 and the Welch Foundation under grant A-1358 (C.M.K.), and the U.S. National Science Foundation under grants PHY-0354572 and PHY-0456890 and the NASA-Arkansas Space Grants Consortium Award ASU15154 (B.A.L.).
[1] I. Bombaci and U. Lombardo, Phys. Rev. C 44, 1892 (1991).

[2] S. Ulrych and H. Müther, Phys. Rev. C 56, 1788 (1997).

[3] C. B. Das, S. Das Gupta, C. Gale, and B. A. Li, Phys. Rev. C 67, 034611 (2003).

[4] B. A. Li, Phys. Rev. C 69, 064602 (2004).

[5] B. A. Li, C. B. Das, S. Das Gupta, and C. Gale, Phys. Rev. C 69, 011603(R) (2004); Nucl. Phys. A735, 563 (2004).

[6] L. W. Chen, C. M. Ko, and B. A. Li, Phys. Rev. C 69, 054606 (2004).

[7] J. Rizzo, M. Colonna, M. Di Toro, and V. Greco, Nucl. Phys. A732, 202 (2004).

[8] E. N. E. van Dalen, C. Fuchs, and A. Faessler, Nucl. Phys. A744, 227 (2004).

[9] Z. Y. Ma, J. Rong, B. Q. Chen, Z. Y. Zhu, and H. Q. Song, Phys. Lett. B604, 170 (2004).

[10] L. W. Chen, C. M. Ko, and B. A. Li, Phys. Rev. Lett. 94, 032701 (2005).

[11] B. Behera, T. R. Routray, A. Pradhan, S. K. Patra, and P. K. Sahu, Nucl. Phys. A753, 367 (2005).

[12] V. Baran, M. Colonna, V. Greco, and M. Di Toro, Phys. Rep. 410, 335 (2005).
[13] F. Sammarruca, W. Barredo, and P. Krastev, Phys. Rev. C 71, 064306 (2005).

[14] W. Zuo, L. G. Cao, B. A. Li, U. Lombardo, and C. W. Shen, Phys. Rev. C 72, 014005 (2005).

[15] E. N. E. van Dalen, C. Fuchs, and A. Faessler, Phys. Rev. Lett. 95, 022302 (2005)

[16] J. Rizzo, M. Colonna, and M. Di Toro, Phys. Rev. C (2005), in press; nucl-th/0508008.

[17] L. G. Arnold, B. C. Clark, and R. L. Mercer, Phys. Rev. C 19, 917 (1979).

[18] J. A. McNeil, J. R. Shepard, and S. J. Wallace, Phys. Rev. Lett. 50, 1439 (1983).

[19] J. R. Shepard, J. A. McNeil, and S. J. Wallace, Phys. Rev. Lett. 50, 1443 (1983).

[20] B. C. Clark, S. Hama, R. L. Mercer, L. Ray, and B. D. Serot, Phys. Rev. Lett. 50, 1644 (1983).

[21] L. D. Miller, Phys. Rev. Lett. 51, 1733 (1983).

[22] J. A. McNeil, L. Ray, and S. J. Wallace, Phys. Rev. C 27, 2123 (1983).

[23] B. D. Serot and J. D. Walecka, Adv. Nucl. Phys. 16, 1 (1986); Int. J. Mod. Phys. E 6, 515 (1997). 
[24] G. A. Lalazissis, J. König, and P. Ring, Phys. Rev. C 55, 540 (1997).

[25] C. J. Horowitz and J. Piekarewicz, Phys. Rev. Lett. 86, 5647 (2001); Phys. Rev. C 64, 062802(R) (2001); 66, 055803 (2002).

[26] J. K. Bunta and S. Gmuca, Phys. Rev. C 68, 054318 (2003).

[27] C. J. Horowitz, Phys. Rev. C 31, 1340 (1985).

[28] D. P. Murdock and C. J. Horowitz, Phys. Rev. C 35, 1442 (1987).

[29] J. A. Tjon and S. J. Wallace, Phys. Rev. C 32, 267 (1985); 35, 280 (1987); 36, 1085 (1987).

[30] N. Ottenstein, S. J. Wallace, and J. A. Tjon, Phys. Rev. C 38, 2272 (1988).

[31] K. Kaki and H. Toki, Nucl. Phys. A696, 453 (2001); K. Kaki,
H. Toki, and I. Tanihata, ibid. A724, 99 (2003).

[32] Y. Jin and R. W. Finlay, Phys. Rev. C 47, 1697 (1993).

[33] R. Brockmann, Phys. Rev. C 18, 1510 (1978).

[34] M. Jaminon, C. Mahaux, and P. Rochus, Phys. Rev. C 22, 2027 (1980); Nucl. Phys. A365, 371 (1981).

[35] M. Jaminon and C. Mahaux, Phys. Rev. C 40, 354 (1989).

[36] A. M. Lane, Nucl. Phys. 35, 676 (1962).

[37] C. Fuchs (private communications).

[38] C. M. Ko, Q. Li, and R. Wang, Phys. Rev. Lett. 59, 1084 (1987); C. M. Ko and Q. Li, Phys. Rev. C 37, R2270 (1988); C. M. Ko and G. Q. Li, J. Phys. G 22, 1673 (1996).

[39] T. Maruyama, B. Blättel, W. Cassing, A. Lang, U. Mosel, and K. Weber, Phys. Lett. B297, 228 (1992). 